\title{
Epigenetic silencing of EYA2 in pancreatic adenocarcinomas promotes tumor growth
}

\author{
Audrey Vincent ${ }^{1}$, Seung-Mo Hong ${ }^{1}$, Chaoxin Hu${ }^{1}$, Noriyuki Omura ${ }^{1}$, Angela Young ${ }^{1}$, \\ Haeryoung Kim ${ }^{1}$, Jun $\mathbf{Y u}^{1}$, Spencer Knight ${ }^{1}$, Michael Ayars ${ }^{1}$, Margaret Griffith ${ }^{1}$, \\ Isabelle Van Seuningen ${ }^{4,5,6}$, Anirban Maitra ${ }^{1}$ and Michael Goggins ${ }^{1,2,3}$ \\ ${ }^{1}$ Department of Pathology, the Sol Goldman Pancreatic Cancer Research Center, Johns Hopkins Medical Institutions, Johns \\ Hopkins University, Baltimore, MD, USA \\ 2 Department of Oncology, the Sol Goldman Pancreatic Cancer Research Center, Johns Hopkins Medical Institutions, Johns \\ Hopkins University, Baltimore, MD, USA \\ ${ }^{3}$ Department of Medicine, the Sol Goldman Pancreatic Cancer Research Center, Johns Hopkins Medical Institutions, Johns \\ Hopkins University, Baltimore, MD, USA \\ ${ }^{4}$ Inserm, UMR837, Jean-Pierre Aubert Research Center, Lille Cedex, France, \\ ${ }^{5}$ Université Lille Nord de France, Lille Cedex, France, \\ ${ }^{6}$ Centre Hospitalier Régional et Universitaire de Lille, Lille Cedex, France. \\ Correspondence to: Michael Goggins, email: mgoggins@jhmi.edu
}

Keywords: EYA2, pancreatic cancer, epigenetic

Received: February 14, $2014 \quad$ Accepted: March 20, $2014 \quad$ Published: March 22, 2014

This is an open-access article distributed under the terms of the Creative Commons Attribution License, which permits unrestricted use, distribution, and reproduction in any medium, provided the original author and source are credited.

\section{ABSTRACT:}

To identify potentially important genes dysregulated in pancreatic cancer, we analyzed genome-wide transcriptional analysis of pancreatic cancers and normal pancreatic duct samples and identified the transcriptional coactivator, EYA2 (Drosophila Eyes Absent Homologue-2) as silenced in the majority of pancreatic cancers. We investigated the role of epigenetic mechanisms of EYA2 gene silencing in pancreatic cancers, performed in vitro and in vivo proliferation and migration assays to assess the effect of EYA2 silencing on tumor cell growth and metastasis formation, and expression analysis to identify genes transcriptionally regulated by EYA2. We found loss of tumoral Eya2 expression in 63\% of pancreatic cancers ( $120 / 189$ cases). Silencing of EYA2 expression in pancreatic cancer cell lines correlated with promoter methylation and histone deacetylation and was reversible with DNA methyltransferase and HDAC inhibitors. EYA2 knockdown in pancreatic cancer cell lines increased cell proliferation. Compared to parental pancreatic cancer cells, pancreatic cancers stablyexpressing EYA2 grew more slowly and had fewer metastases in orthotopic models. The transcriptional changes after stable expression of EYA2 in pancreatic cancer cells included induction of genes in the TGFbeta pathway. Epigenetic silencing of EYA2 is a common event in pancreatic cancers and stable expression EYA2 limits the growth and metastases of pancreatic adenocarcinoma.

\section{INTRODUCTION}

Pancreatic cancer is the fourth leading cause of cancer-related death in the United States, with a dismal 5 -year survival rate of $\sim 5 \%$. The majority of patients are diagnosed with advanced-stage disease, contributing to their poor prognosis [1]. There is a need to understand the molecular mechanisms responsible for pancreatic cancer development and progression in order to identify effective therapeutic targets for this disease and whenever possible ensure its early detection. Pancreatic ductal adenocarcinomas develop as a result of clonallyselected genetic events most commonly involving the genes KRAS, CDKN2A, TP53 and SMAD4 [2-6], less commonly $A T M$ and others $[3,5,7,8]$, and for pancreatic ductal adenocarcinomas arising from intraductal 
papillary mucinous neoplasms, most commonly $K R A S$, $C D K N 2 A$, TP53, as well as GNAS and RNF43 [911]. Previous studies have demonstrated that aberrant expression of epigenetically regulated genes contributes to pancreatic cancer development and progression [12-16]. To further identify epigenetically deregulated genes in pancreatic cancers, we compared the published SAGE (Serial analysis of gene expression) profiles of pancreatic ductal adenocarcinomas and normal pancreatic duct cells [3], focusing on silenced genes implicated in cancer progression that had not been reported as silenced in pancreatic cancer. From this analysis we identified Drosophila Eyes Absent Homologue 2 (EYA2) as a commonly silenced gene in pancreatic ductal adenocarcinomas.

Eya2 is normally expressed early during development and is characterized by two independent functions [17]. As a transcriptional coactivator, Eya2 is involved in the regulation of the retinal determination gene network, essential for eye fate specification, which plays an important role in regulating cell death and/or differentiation during development [18]. Additionally, the tyrosine phosphatase activity of Eya2 [18] has been shown to dephosphorylate $\mathrm{H} 2 \mathrm{AX}$, promoting repair and cell survival in the response to DNA damage [19]. Other EYA family members, such as Eyal and Eya3, are required during mammalian organogenesis and through their phosphatase activity regulate genes encoding growth control, signaling and survival [20].

Overexpression of EYA2 has been shown to promote epithelial ovarian tumor growth [21] and breast cancer metastases [22]. On the other hand, enforced expression of Eya2 has been shown to trigger apoptosis in IL-3-dependant myeloid cells [23], and EYA2 has been found to be aberrantly hypermethylated in most colorectal neoplasms [24], indicating the potential for EYA2 promoter methylation as a marker of tumorigenesis. Against this background, we evaluated the expression of Eya2 in normal pancreas and in pancreatic cancer tissues and cell lines, examined the methylation and histone acetylation status of its promoter and determined the consequences of stably expressing EYA2 in pancreatic cancer cells including effects on tumor growth and metastases in an orthotopic model and effects on gene expression.

\section{RESULTS}

\section{Loss of EYA2 expression in pancreatic cancer}

Bioinformatic analysis of our Serial Analysis of Gene Expression data [3, 25] revealed EYA2 mRNA as underexpressed in pancreatic cancers compared to pancreatic normal duct cells and HPDE, an immortalized non-neoplastic human pancreatic ductal epithelial line. Several hundred genes have been identified as silenced in pancreatic cancers by global gene expression analysis in prior studies [25, 26], but we focused on EYA2 because of its putative functions and because it has not been recognized previously as underexpressed in pancreatic cancer. To confirm the SAGE data, we performed quantitative PCR analysis on HPDE and nine pancreatic cancer cell lines i.e. Panc215, Panc2.5, Panc2.8, Panc3.014, AsPC-1, BxPC-3, MIA PaCa2, Panc1 and Su8686. We found a 5-fold and a 7.8-fold decrease of EYA2 expression in Panc 215 and BxPC-3 cell lines compared to HPDE and very low (Panc2.8, Panc1) or virtually no expression in the seven other cell lines studied (Figure 1A). We then examined the expression of Eya2 protein in 189 primary pancreatic adenocarcinomas and adjacent normal and non-neoplastic pancreas by performing immunohistochemistry on tissue microarrays (Figure 1B-1E). Normal pancreas expression was localized to both the cytoplasm and nucleus but predominantly cytoplasmic (consistent with its phosphatase activity) with some cells displaying only cytoplasmic labeling. Complete loss of Eya2 protein expression was observed in the tumor cells of $63.5 \%$ of primary pancreatic adenocarcinomas (120 of 189 cases), while expression of Eya2 was found in normal ductal cells of $99.5 \%$ of cases. In addition to complete loss of expression, some pancreatic cancers retained only nuclear expression. We did not observe any pancreatic cancers with overexpression relative to normal

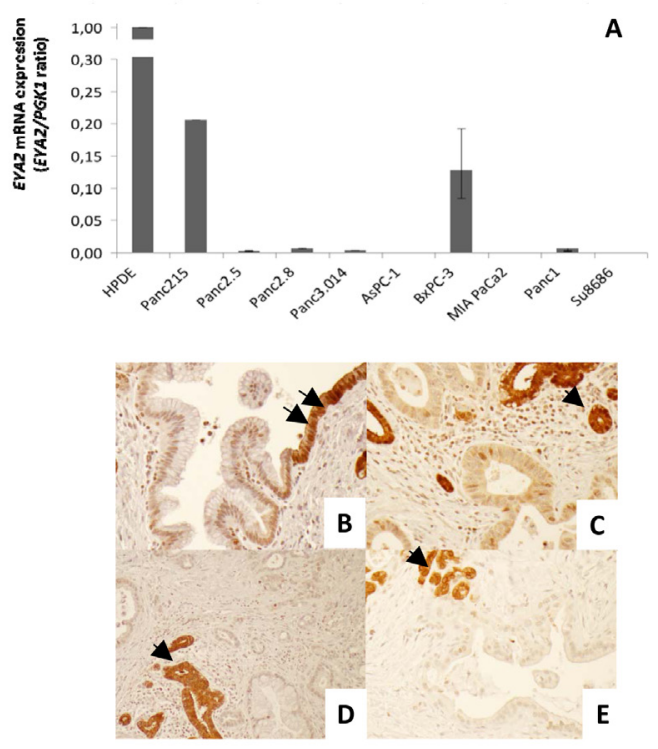

Figure 1:(A) EYA2 expression by real-time PCR in normal HPDE cells and nine pancreatic cancer cell lines. (B-E) Representative figures of immunohistochemical staining of EYA2 with tissue microarrays. (B) Transition from normal ductal epithelium (arrows) to PanIN1. PanIN1 shows a decreased EYA2 expression. (C-E) Pancreatic ductal adenocarcinomas show a decrease or complete loss of EYA2 expression compared to entrapped normal ductal or acinar cells (arrows). Magnification: 10X. 
B

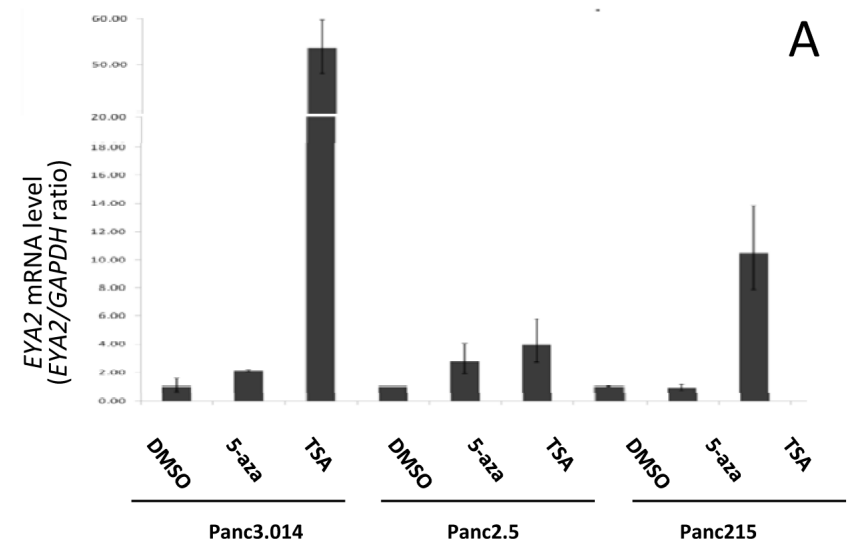

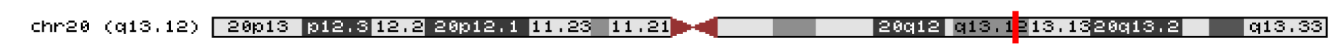
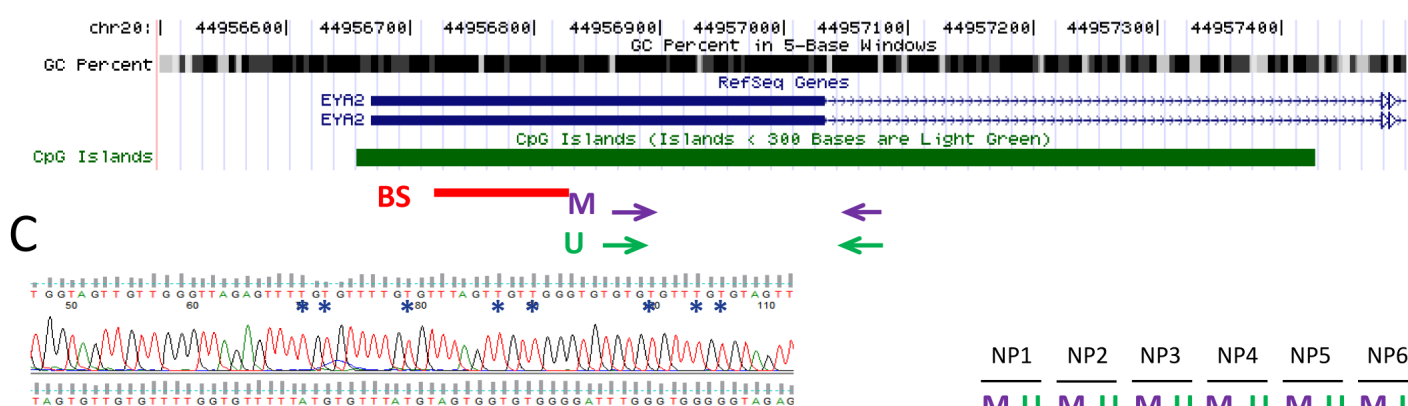

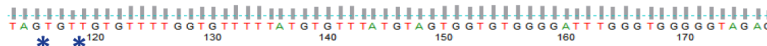

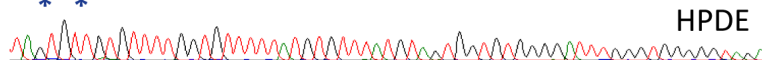

Normal

NP1 NP2 NP3 NP4 NP5 NP6

$\overline{M U} \overline{M U} \bar{M} \bar{M} \bar{M} \cup \bar{M}$ Pancreas

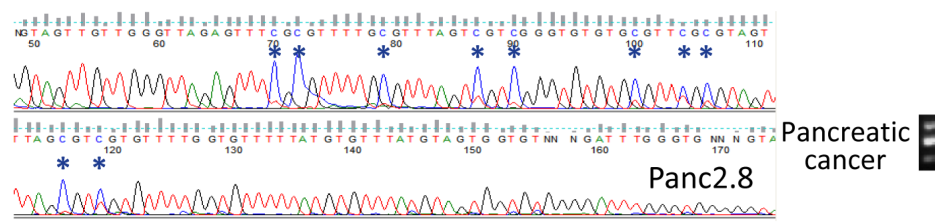

$\frac{P C 1}{M U} \frac{P C 2}{M U} \frac{P C 3}{M U} \frac{P C 4}{M U} \frac{P C 5}{M U} \frac{P C 6}{M U}$

$M U M U M U M U M U M U$

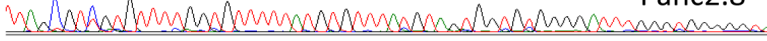

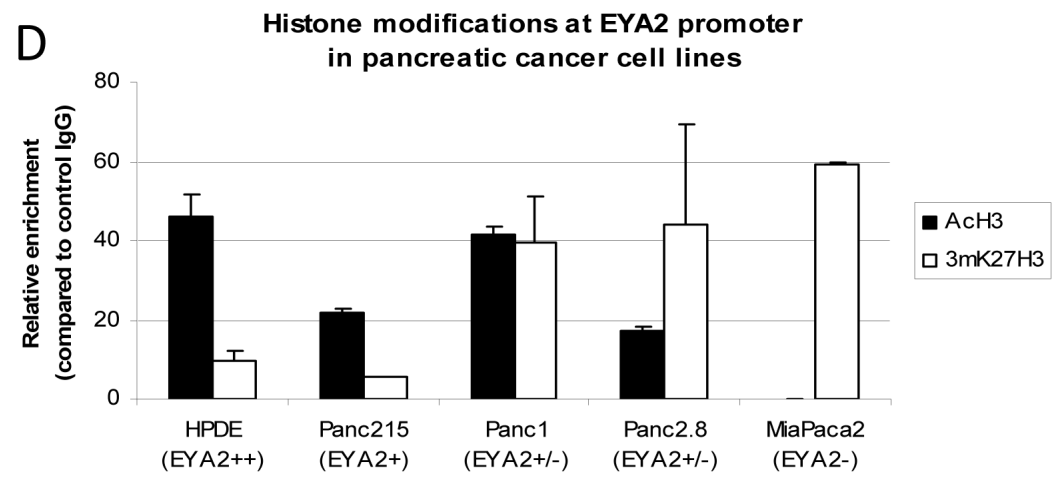

Figure 2: (A) Cells were treated with either 5-Aza-2'-deoxycytidine (5-Aza, $5 \mu \mathrm{M}, 72 \mathrm{hrs}$ ), trichostatin A (TSA, $0.3 \mu \mathrm{M}, 24 \mathrm{hrs}$ ) or DMSO prior to RNA extraction and analysis of EYA2 expression by real-time PCR. $\beta$-actin was used as an internal control for gene expression. (B) Chromosomal localization of EYA2 gene as represented by the UCSC Genome Browser. The GC percent in 5-base windows is shown for this region. The $\mathrm{CpG}$ island spanning the first exon (thick horizontal blue line) and the first part of the first intron (thin horizontal arrowed blue line) is represented in green. Horizontal red bar represents the region studied by bisulfite sequencing (BS). Purple and green arrows represent the primers used for MSP studies (M: Methylated, U: Unmethylated). (C) Representative bisulfite sequencing and MSP in normal pancreatic tissues (upper panels) and in primary pancreatic adenocarcinoma tissues (lower panels). (D) Chromatin immunoprecipitation was performed with specific antibodies against acetylated histone $\mathrm{H} 3(\mathrm{AcH} 3)$ and methylated $\mathrm{K} 27$ of histone $\mathrm{H} 3$ (mK27H3) in the Human Pancreatic Ductal Epithelial cell line HPDE and four pancreatic cancer cell lines harboring different levels of EYA2 expression (Panc215, Panc1, Panc2.8 and MiaPaca2). Enrichment of each histone modification at EYA2 promoter was assessed by qPCR and reported to the control (Normal rabbit $\mathrm{IgG}$ ). 
pancreas. Patients with tumoral loss of Eya2 expression had significantly worse survival (median survival, 17.2 months) compared to patients whose cancers retained Eya2 expression (24.5 months, $\mathrm{P}=0.03$ ), but Eya2 loss was not an independent predictor of survival when other factors associated with outcome (such as stage, differentiation, node status) were considered in a multivariate model (data not shown).

\section{EYA2 is epigenetically silenced in pancreatic cancer cell lines}

Since aberrant methylation is a common mechanism of gene silencing in pancreatic cancer [13], and EYA2 has been shown to be hypermethylated in colon cancers [24], we investigated the role of epigenetic

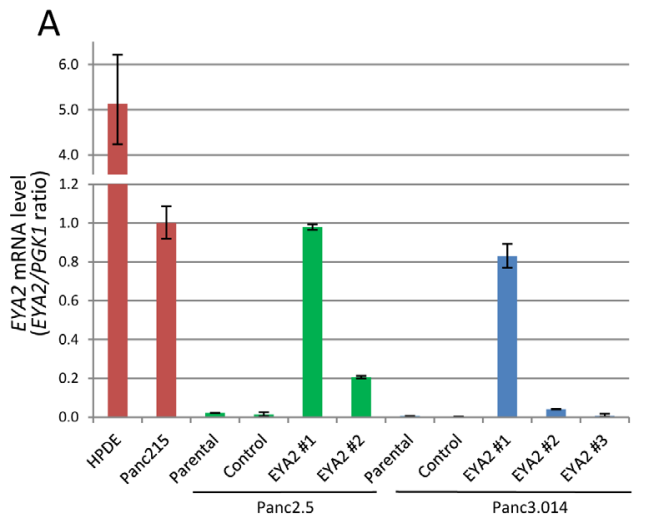

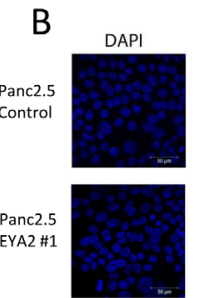
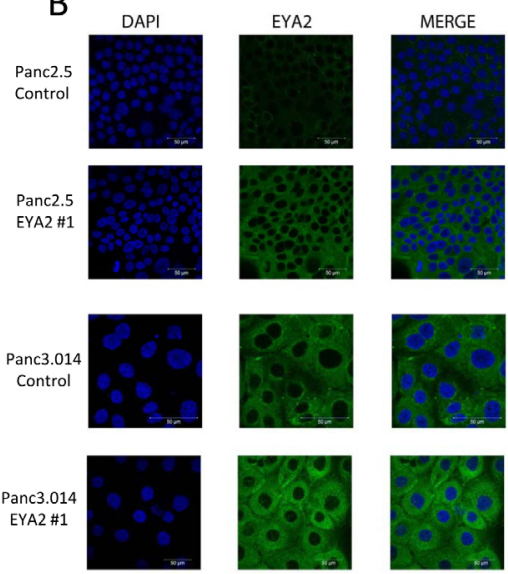

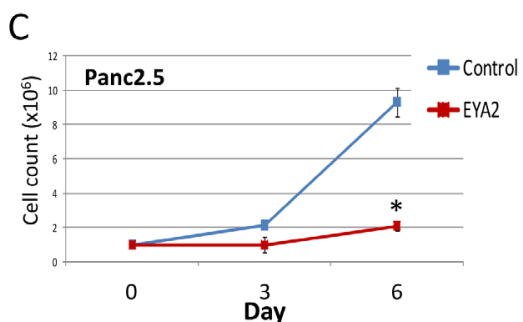

D

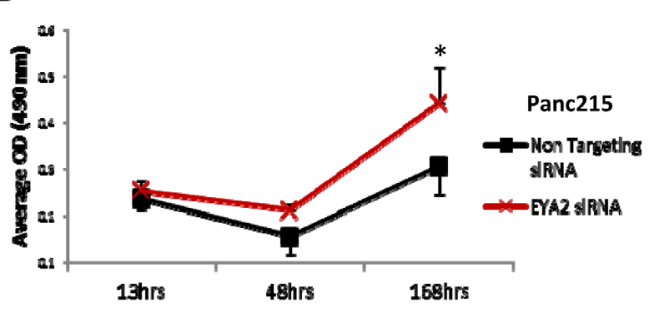

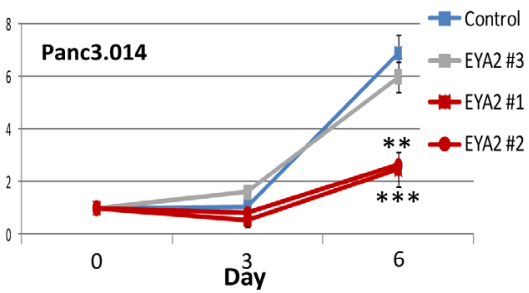
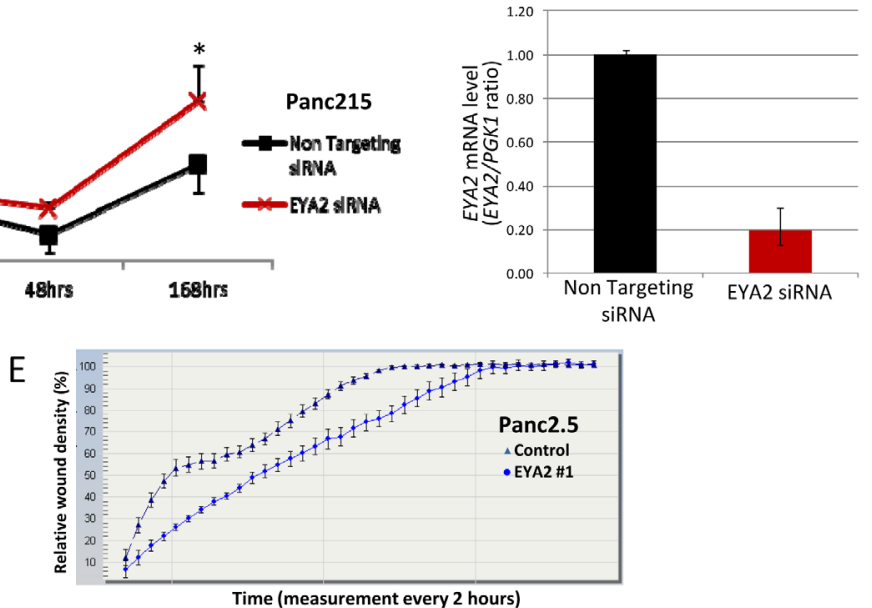

SiRNA

Figure 3: (A) Bar graph indicating the level of EYA2 mRNA expression in positive controls (normal HPDE cells and Panc215 cancer cells), non-transfected cancer cell lines (Panc2.5 and Panc3.014, parental), cells transfected with an empty vector (Panc2.5 and Panc3.014, control) and cells transfected with an EYA2 expressing vector (Panc2.5 EYA2 \#1 and \#2 and Panc3.014 \#1, \#2 and \#3). (B) Confocal microscopy showing EYA2 localization and expression in Panc2.5 and Panc3.014 EYA4 expressing and control clones. (C) Proliferation curves of Panc2.5 and Panc3.014 control cells (blue curves) compared to EYA2 expressing stable transfectants (red curves) and EYA2 non-expressing stable transfectant (grey curve). $1 \times 10^{5}$ cells were plated in 24-well plates and viable cells were then counted after three and six days. (D) Panc215 cancer cells were transfected with either a non-targeting siRNA or a siRNA targeting EYA2 24hrs after seeding. Cell proliferation was assessed by measuring absorbance at $490 \mathrm{~nm}$ after incubation of the cells with Cell Titer 96® Aqueous One Solution Reagent (Promega). *, P $<0.05$; **; P $<0.01 ; * * * . P<0.0001$. EYA2 specific knockdown was assessed by qRT-PCR. (E) Migration assay. Wound density was assessed every two hours for $72 \mathrm{~h}$ using the Incucyte Live-Cell Imaging System 
mechanisms in EYA2 silencing. We first treated two EYA2 non-expressing pancreatic cancer cell lines with the demethylating agent 5-aza-2'-deoxycytidine (5$\mathrm{azaC}$ ) and the histone deacetylase inhibitor, Trichostatin A (TSA). We found that 5-azaC increased EYA2 mRNA expression in Panc3.014 and in Panc2.5, respectively by 2.1 - and 2.8-fold, while TSA increased EYA2 mRNA expression by 53.6 and 4.0-fold, respectively (Figure 2A). Treatment of the EYA2-expressing cell line Panc215 with 5-azaC had no significant effect on EYA2 mRNA expression and treatment with TSA induced a 10.5-fold increase of EYA2 mRNA expression (Figure 2A). We then examined $E Y A 2$ promoter methylation by bisulfite sequencing in normal HPDE cells and five pancreatic

A
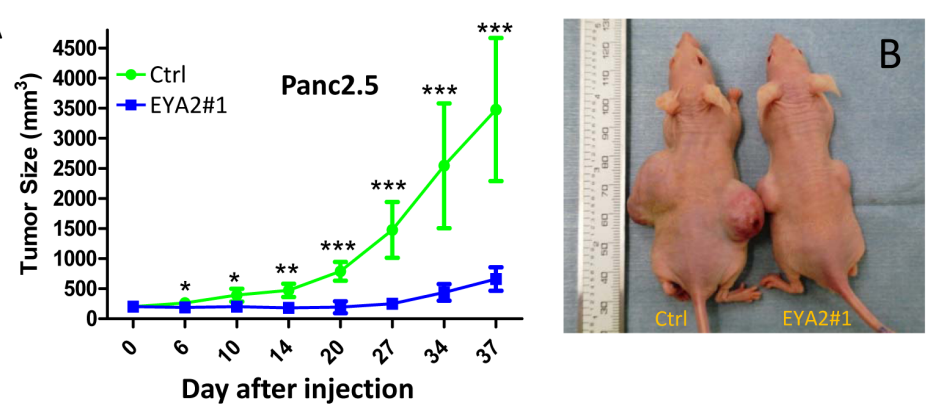

C
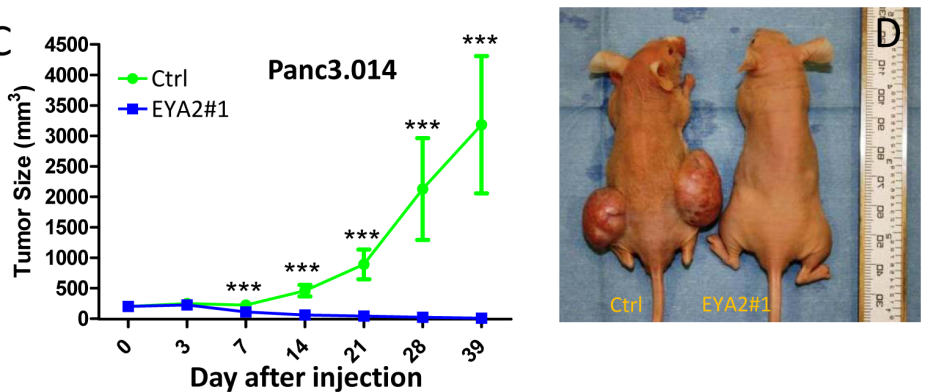

E

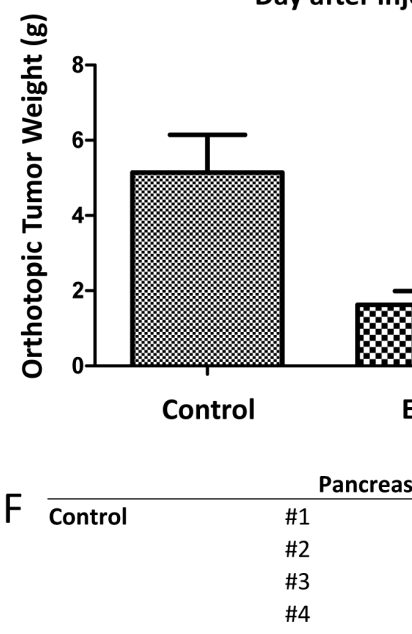

EYA2

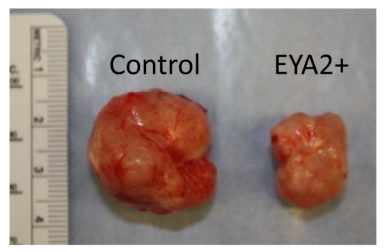

EYA2

overexpressing

$\begin{array}{ll}\# 1 & 0 \\ \# 2 & 0 \\ \# 3 & 0 \\ \# 4 & 0\end{array}$

Lymph

$\begin{array}{rrrr}\text { nodes } & \text { Lung Intestine } & \text { Total } \\ 2 & 3 & 4 & 12\end{array}$

$\begin{array}{rrrr}2 & 3 & 12 & 9\end{array}$

$\begin{array}{llll}5 & 0 & 5 & 11 \\ 8 & 0 & 7 & 16\end{array}$

Av. $=15$

$\begin{array}{lll}\# 2 & 0 \\ \# 3 & 0 \\ \# 4 & 0\end{array}$

$\begin{array}{rrrr}0 & 0 & 4 & 4 \\ 0 & 0 & 1 & 1 \\ 0 & 0 & 2 & 2 \\ 0 & 0 & 0 & 0 \\ & & & A v .=1.75 \\ & & & p \text {-value }=\mathbf{0 . 0 0 3 7}\end{array}$

Figure 4: (A-D) Panc2.5 and Panc3.014 control and EYA2-overexpressing cells were used to perform subcutaneous xenografts in Nude mice. For each stable clone, five mice were xenografted and data represent Mean $\pm \mathrm{SD}$. *, $\mathrm{P}<0.05 ; * * ; \mathrm{P}<0.01 ; * * *$. $\mathrm{P}<0.0001$. Tumor size is significantly smaller in mice xenografted with EYA2-overexpressing cells than in control cells. (E-F) Tumours obtained from subcutaneous xenografts of Panc2.5 control and EYA2-overexpressing cells were used to perform orthotopic xenografts in eight additional Nude mice. (E) Tumour weight 77 days after orthotopic implantation. (F) Total number of macroscopically observed metastases in mice xenografted with EYA2-overexpressing cells compared to control cells. The primary tumour in the pancreas was excluded from counting and does not appear in the table. 
cancer cell lines. We found the EYA2 CpG island (Figure 2B) unmethylated in HPDE and the EYA2-expressing cancer cell line, Panc215, but it was methylated at most or all $\mathrm{CpGs}$ sequenced in the non-expressing Panc2.8 and A38-5 cell lines, respectively (Figure 2C), and remained unmethylated in the non-expressing Panc2.5 and Panc3.014 cells. We then used methylation-specific PCR (MSP) to assess EYA2 promoter methylation in 53 pancreatic cancers ( 9 cell lines, 23 primary pancreatic cancer tissues and 21 xenografts of primary pancreatic cancers) and 58 normal pancreatic tissues. Complete DNA methylation of the EYA2 promoter region amplified by MSP was identified in Panc2.8, Panc215, Panc2.5, and Panc3.014 cell lines, partial methylation in AsPC1 and only unmethylated templates in EYA2-expressing BxPC3 cancer cells. We found no evidence of promoter methylation in the non-expressing MiaPaca2, Panc1 and
Su8686 cell lines suggesting that promoter methylation was not always required for loss of EYA2 expression. Overall, methylation of the EYA2 promoter was found in $22.7 \%$ of pancreatic adenocarcinomas versus $6.9 \%$ of normal pancreatic tissues $(p=0.019)$ (Figure $2 C)$. Since the histone deacetylation inhibitor TSA could induce EYA2 expression without 5 -aza-dC in some cell lines, we further examined histone acetylation for its role in regulating EYA2 expression (Figure 2A). Analysis of histone marks in pancreatic cancer cell lines by chromatin immunoprecipitation indicated a correlation between EYA2 silencing and histone $\mathrm{H} 3$ deacetylation of the $E Y A 2$ promoter (Figure 2D) where non-expressing cells showed a significant enrichment of the inactivating histone mark, 3mK27H3, while high-expressing cells showed a significant enrichment in acetylation of histone $\mathrm{H} 3$.
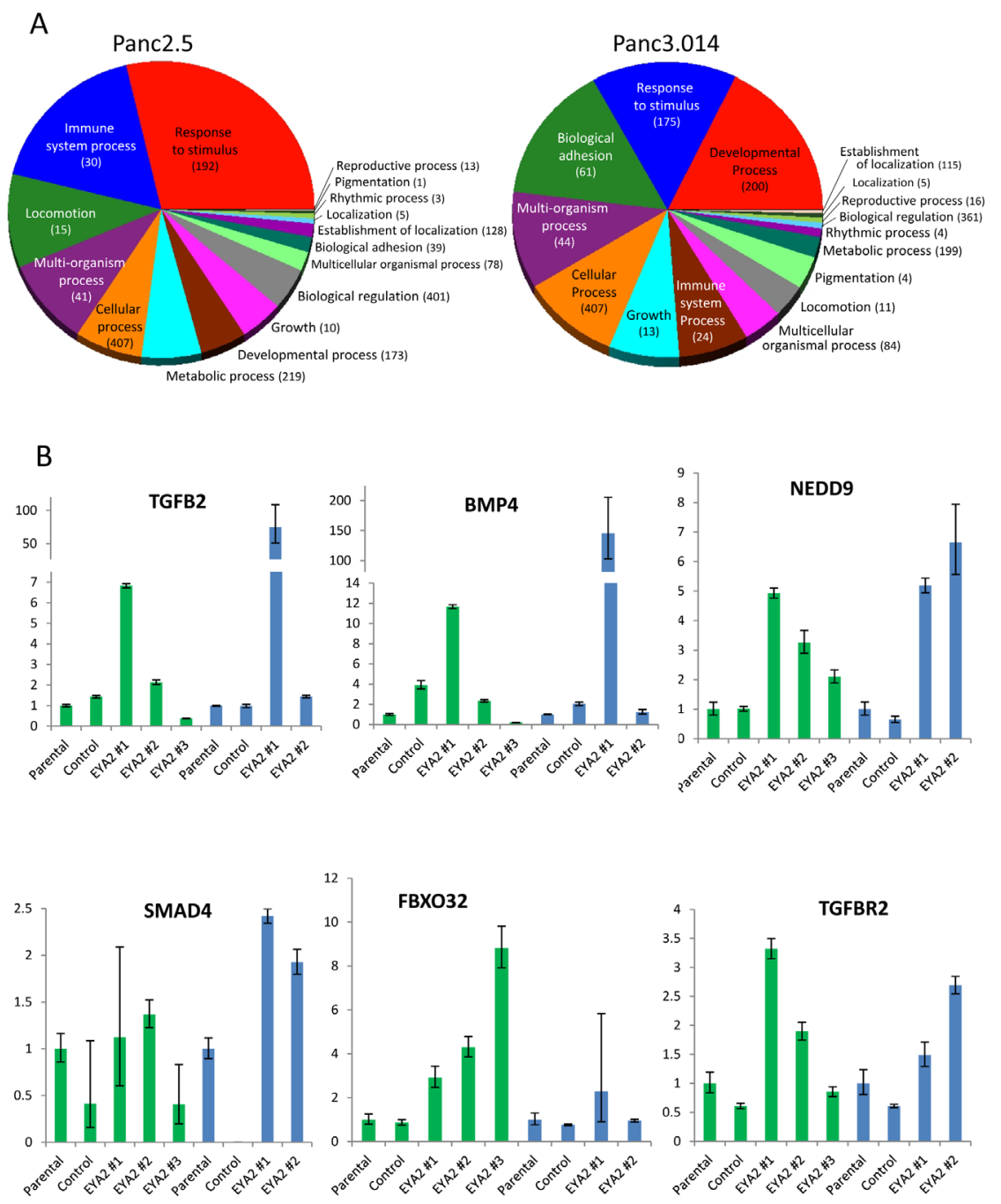

Figure 5: (A) Summary of differentially expressed genes in Panc2.5 and Panc3.014 EYA2-overexpressing clones compared to control clones. Statistical analysis of gene expression array data as well as Gene Ontology classification were performed with Partek Genomic Suite 6.4 software. (B) Quantitative RT-PCR showing the expression profile of genes from the TGFbeta pathway in EYA2-overexpressing clones compared to control clones and untransfected cells (Parental) in Panc2.5 (green bars) and Panc3.014 (blue bars) cells. Bands were quantified by densitometry. 


\section{EYA2 overexpression decreases pancreatic cancer cell proliferation in vitro}

To investigate the function of EYA2 in pancreatic cells, we established stable EYA2-overexpressing clones from Panc2.5 and Panc3.014 pancreatic cancer lines using the pcDNA6.2/cLumio-DEST containing the EYA2 transcript. Stable transfectants showed a significant overexpression of EYA2 transcripts (Figure $3 \mathrm{~A})$. For subsequent experiments, we selected stable clones in which EYA2 expression was comparable to the naturally expressing cancer cell line Panc215. Using immunocytofluorescence, we found that EYA2 protein was also overexpressed in these stable transfectants and showed perinuclear localization in the cells (Figure 3B).

We then compared cell proliferation in the stable transfectants expressing EYA2, to control transfected cells (empty pcDNA6.2/cLumio-DEST). Our results showed that EYA2-expressing Panc2.5 cells proliferated 4.4 times slower than control Panc2.5 cells (Figure 3C). Similarly, Panc3.014 EYA2-expressing clones \#1 and \#2 showed a 2.78 and 2.61-fold decrease in cell growth compared to control cells, respectively (Figure 3C). Panc3.014 stable clone \#3, which expressed the same level of EYA2 as Panc3.014 control cells, showed a similar proliferation rate compared to Panc3.014 control cells (Figure 3C). Conversely, we used a specific siRNA to inhibit EYA2 expression in Panc215 cancer cells which yielded a 5.1fold decrease of EYA2 expression (Figure 3D) associated with a significant increase in cell proliferation $(\mathrm{P}=0.0015$, Figure 3D).

We also compared cell migration in the stable transfectants expressing EYA2, to control transfected cells. Our results showed that EYA2-expressing Panc2.5 cells migrated 1.3 times slower than control Panc2.5 cells (Figure 3E).

\section{EYA2 overexpression prevents cell growth and metastasis in vivo}

We further analyzed the role of EYA2 on tumor growth in vivo using an immunodeficient mouse model. Thirty-seven days after subcutaneous cell injection EYA2-overexpressing Panc 2.5 cells showed a significant decrease in tumor size (7-fold, $\mathrm{P}<0.0001$, Figure 4A and 4B) compared to Panc2.5 control cells. Similarly, EYA2-overexpressing Panc3.014 cells totally lost their potential to form a tumor in vivo (Figure 4C and 4D). To further evaluate the effects of EYA2 on tumor growth and on metastases, we implanted $1 \mathrm{~mm}^{3}$ subcutaneous tumors from Panc2.5 stable transfectant and control cells into the pancreas as orthotopic xenografts in the same immunodeficient mouse model. After 11 weeks, we found that EYA2-overexpressing Panc2.5 cells showed a significant decrease in tumor weight (3-fold,
$\mathrm{P}<0.0001$, Figure 4E) compared to control Panc2.5 cells. Additionally, we checked macroscopically for the presence of visible metastasis in lymph nodes, liver, lung and intestine as well as evidence of tumor cells in the pancreas, distant from the site of orthotopic grafting. We were not able to observe metastasis in the lungs, liver and lymph nodes of mice xenografted with EYA2-overexpressing tumors, while we observed several metastatic tumors in each mouse xenografted with control Panc2.5 tumors (Mean $\pm \mathrm{SD}, 5.5 \pm 4.4$ tumors, $\mathrm{P}=0.025$ ).

\section{EYA2 overexpression in pancreatic cancer cells modulates gene expression}

To further understand the role of EYA2 on tumor growth and metastasis, we compared the transcriptomes of EYA2-overexpressing vs. control Panc2.5 and Panc3.014 cells (the same clones that were xenografted) using Affymetrix ST1.0 Exon Arrays. We identified one hundred and sixty genes differentially expressed by $>2$ fold in both of the EYA2-expressing cells relative to their corresponding control cells (stable empty vectorexpressing Panc2.5 and Panc3.014 cells) (Supplemental Table 1), with additional genes showing expression changes in one of the two cell lines (data not shown). One hundred eighteen of these 160 genes contained a putative binding site for the EYA2 transcription factor in their promoter (Supplemental Table 1). We attempted to perform Chip analysis to identify EYA2 binding sites but available commercial antibodies did not enrich for putative EYA2 targets tested (data not shown). This list of genes induced by stable EYA2 expression in these cells included those responsible for growth and/or developmental processes (such as IGF2, PTGS2, DZIP1, and NOTCH3), response to injury (TFF1), the stem cell marker (CD133 (PROM1), and genes related to adhesion (such as ANTXR1, COL12A1, ITGB7, LAMA4 and NID1) and genes in the TGF beta signaling pathway or its downstream targets, either in Panc2.5 (TGFBR2, FBXO32) or Panc3.014 (SMAD4, CEACAM5) cells or in both lines (TGFB2, BMP4, THBS1, EID2, and NEDD9) [27, 28] (Figure 5A). We examined the expression of seven of these genes by quantitative RT-PCR in the cell clones that were subcutaneously xenografted in nude mice as well as in one additional EYA2-overexpressing clone for each cell line (Figure 5B and Supplemental Figure 1). Additionally, we demonstrated overexpression of TGFBR2 protein by western blot in both EYA2-overexpressing clones (Supplemental Figure 2). We also observed a modest decrease in TGFBR2 receptor phosphorylation in the Panc2.5-EYA2-overexpressing cells compared to control cells suggesting that the phosphatase activity of EYA2 reduced the proportion of TGFBR2 phosphorylation in EYA2-expressing cells (Supplemental Figure 2). 


\section{DISCUSSION}

Here, we demonstrate that the majority of pancreatic cancers lose expression of the developmental transcription factor and phosphatase, EYA2. This loss of expression is associated with poorer patient survival and under expression is evident early during pancreatic tumorigenesis, even at the PanIN-1 stage. We also find evidence for epigenetic regulation of EYA2 in pancreatic cancers with both aberrant DNA methylation and chromatin marks in different pancreatic cancers. Furthermore, EYA2 expression had a dramatic reduction in tumor growth in vivo both in subcutaneous and in orthotopic models, in two different pancreatic cancer cell lines and in multiple clones. Indeed, no tumor growth was observed when EYA2-expressing Panc3.014 cells were xenografted. Furthermore, orthotopically implanted EYA2-expressing pancreatic cancers had many fewer metastases, beyond what would be expected by the reduced size of the primary tumor. Knockdown of EYA2 in EYA2-expressing pancreatic cancer cells resulted in an increase in cell proliferation and stably-expressing EYA2 pancreatic cancer cells had reduced cell migration.

Eya2 plays an important role in numerous species in regulating cell death and differentiation and is expressed throughout development [29-31] and influences human developmental processes [32].

In contrast to the tumor suppressive functions of EYA2 in pancreatic cancers, Zhang and collaborators [21] found that EYA2 is up-regulated in epithelial ovarian cancer, promotes tumor growth and its overexpression is associated with short overall survival and Farabaugh et al found EYA2 had prometastatic functions in breast cancer [22]. These data suggest that EYA2 has organ specific functions, perhaps depending on its cellular localization, and underlines the complexity of its double functions as a phosphatase and as transcription coactivator during differentiation and cell signaling processes [18]. These findings suggest that EYA2 may function as an oncogene or tumor suppressor depending on the cell type, an observation that has been observed for several other genes such as NOTCH1 [33]. Furthermore, it has been shown previously that EYA-induced cell death signals override survival factors and has many features typical of apoptosis [23]. Furthermore, prior studies have also found that Eya mutants overproliferate [18].

Consistent with its role as a transcription factor, we identified many genes that were either upregulated or downregulated in the EYA2-expressing pancreatic cancer cells, many of which were involved in developmental processes, cell growth and adhesion. Further work is needed to determine if these expression changes represent direct transcriptional effects or indirect effects of inducing EYA2 expression. The effects of stably-expressing EYA2 in pancreatic cancer cells we observed on TGF beta pathway gene expression may be of particular interest since the TGF beta signaling pathway is disrupted in many pancreatic cancers and as evidenced by the inactivating mutations in DPC4 in $\sim 55 \%$ of pancreatic cancers and the occasional genetic inactivation of other pathway components (TGFBR2, TGFBR1, BMPR2)[3, 5, 34, 35]. Interestingly, EYA2 has recently been reported to influence TGF beta signaling in breast cancer cells [22], but in contrast to our findings in pancreatic cancer, these authors found that EYA2 functioned as a promoter of growth and metastases and a potential therapeutic target for inhibition. Our results indicate that EYA2 not only influences transcription of TGF beta pathway members but also affects phosphorylation of TGFBR2suggesting a dual role of EYA2 in the pancreas.

In summary, we find that silencing of EYA2 in the majority of pancreatic cancers; that such silencing is associated with poor outcome with pancreatic cancer; that EYA2 can suppress tumor growth in orthotopic models of pancreatic cancer, and that EYA2 signaling alters the expression of many genes involved in growth and development.

\section{MATERIAL AND METHODS}

\section{Cell lines and tissue samples}

Human pancreatic cancer cell lines Panc2.5 (Pa21C), Panc2.8 and Panc3.014 (Pa28C) were cultured as previously described [3]. HPDE cells were generously provided by Dr Ming-Sound Tsao (University of Toronto, Ontario, Canada).

EYA2-overexpressing clones were established by stably transfecting the pcDNA6.2/cLumio-DEST vector containing the EYA2 transcript in Panc2.5 and Panc3.014 pancreatic cancer lines. The Gateway cloning system (Invitrogen) was used to clone the EYA2 transcript variant ENSG00000064655 in the pcDNA6.2/cLumio-DEST vector.

Discarded frozen normal and neoplastic tissues were obtained from patients who had undergone pancreatic resection for pancreatic adenocarcinoma or pancreatic neuroendocrine neoplasm at Johns Hopkins Hospital. In addition, tissue microarrays (TMAs) of formalinfixed paraffin-embedded tissues were retrieved from 189 patients who underwent surgical resection at our institution. Specimens were collected and analyzed with the approval of the Johns Hopkins Committee for Clinical Investigation.

\section{Immunohistochemistry}

Immunohistochemical labeling was performed using HRP EnVision ${ }^{+}$System (DAKO Corp.) on TMA slides as previously described [36]. Deparaffinized slides were 
subjected to heat-induced epitope retrieval using a steamer and DAKO Target retrieval solution ( $\mathrm{pH}$ 6.0-6.2; DAKO Corp.). Slides were incubated with goat polyclonal antihuman EYA2 (Santa Cruz, clone N16, catalog number is 15097) diluted to 1:200. A semi-quantitative analysis of immunostaining was performed, quantifying both the percentage and intensity of labelled ductal cells. The intensity of immunolabeling of individual cells was scored on a scale from 0 (no staining) to 4 (strongest intensity). The percentage of labelled cells at each intensity level was multiplied by the corresponding intensity value to obtain an immunolabeling score. For survival analysis, immunolabeling was categorized as present or absent.

\section{Confocal microscopy}

Confocal microscopy was performed on Panc2.5and Panc3.014-EYA2-expressing and control clones grown on Lab-Tek Chamber Slide permanox (Nunc) as described previously [37]. Cells were incubated overnight with EYA2-specific antibody. Slides were mounted in mounting medium containing DAPI and visualized with a Zeiss LSM 710 confocal microscope (Carl Zeiss Microscopy); images were captured and analysed with the Zeiss Efficient Navigation software (ZEN, Carl Zeiss Microscopy). Cell fluorescence was measured with Image J software as previously described [38].

\section{Treatment with 5-aza-2'-deoxycytidine (5-aza-dC) and Trichostatin A (TSA)}

Cells were treated with 5-aza-dC (Sigma Chemical Co) at $1 \mu \mathrm{mol} / \mathrm{L}$ for 4 days and/or $1 \mu \mathrm{mol} / \mathrm{L}$ of TSA for 24 hours as previously described [39].

\section{Quantitative reverse-transcriptase PCR (qRT- PCR)}

Total RNA from cell lines was extracted using mirVana miRNA Isolation Kit (Ambion, Austin, TX) following the manufacturer's protocol and treated with DNA-free kit (Ambion). One $\mu \mathrm{g}$ of total RNA were reverse transcribed using Superscript ${ }^{\circledR}$ III Reverse Transcriptase and random hexamers (Invitrogen Life Technologies; Carlsbad, CA) for qRT-PCR. EYA2 cDNAs were quantified using SYBR Green PCR Master Mix for SYBR green I (Applied Biosystems, Foster City, CA). PCR was performed on an ABI7300 real-time thermocycler. Primers for EYA2 were Fwd: 5'-GGACAATGAGATTGAGCGTGT-3' and Rev: 5'-ATGTCCCCGTGAGTAAGGAGT-3'. The housekeeping gene $A C T B$ was used as a reference.

\section{Bisulfite modified sequencing and Methylation specific PCR}

The methylation status of EYA2 promoter was determined by bisulfite modified sequencing (BMS) and methylation-specific PCR (MSP) as previously described [40]. For BMS, primers were Fwd 5'-AGGAGGTTGGGTTTTGGTT-3' and Rev 5'ATA AACAACTCCCCCC-3'. For MSP, DNA treated with SssI methylase (New England Biochemicals) and whole-genome amplified DNA (REPLI-g Mini Kit, Qiagen) were used as controls for methylated and unmethylated DNA, respectively. Primers were U: Fwd 5'-GGGAGGAGAAGGGGTTGGTTTTTTTG-3' and Rev 5'-CCTAAAATAAACACCACTAACAATA CTCACCA-3'; M: Fwd 5'-TTTCGGCGTAGGTAGTAGTCGC-3' and Rev 5'-GACCTAAAATAAACGCCGCTAACGA-3'.

\section{Chromatin Immunoprecipitation (ChIP)}

ChIP was performed as previously described [41]. Briefly, cells were treated with $1 \%(\mathrm{v} / \mathrm{v})$ formaldehyde and cross-links were quenched with glycine. Cells were rinsed with ice-cold PBS with protease inhibitors (Roche Applied Science), scraped and collected by centrifugation, before being resuspended in lysis buffer plus protease inhibitors. Chromatin was sheared with the Bioruptor system (Diagenode) to generate DNA fragments with an average size of $\sim 200-400 \mathrm{bp}$. Antibodies to the repressive mark $\mathrm{H} 3 \mathrm{~K} 27 \mathrm{~m} 3$ and the active mark, acetylated $\mathrm{H} 3$, or normal IgG as a control were used. DNA was PCR amplified with primers targeting EYA2 promoter (Fwd: CCTGCGCCTCTTTCTGGCACT and Rev: TCTGCCCTTGTGCCTTCCTGG).

\section{Small Interfering RNA Transfection}

A siRNA targeting EYA2 (ON-TARGETplus SMARTpool EYA2, L-017233) and a non-targeting control siRNA were obtained from Dharmacon RNAi Technologies (Lafayette, CO). $1 \times 10^{5}$ cells/well of Panc 215 cells, were seeded in a 24 well plate and incubated for 24 hrs. Cells were transfected with EYA2 siRNA or control siRNA (100 nmol/L) using DharmaFECT4 transfection reagent, incubated either for $48 \mathrm{hr}$ or 7 days before cell counting. Specific EYA2 knockdown was assessed by qRTPCR as described above.

\section{Proliferation and migration Assays}

For proliferation assays, 4,000 cells/well were seeded onto 96-well plates and incubated overnight. Cell proliferation was quantified either by adding $20 \mu \mathrm{l}$ of 
CellTiter $\mathrm{AQ}_{\text {ueous }}$ One Solution (Promega) into each well containing $100 \mu \mathrm{l}$ culture medium, and incubated for $2 \mathrm{~h}$ at $37{ }^{\circ} \mathrm{C}$ before absorbance measurement at $490 \mathrm{~nm}$ or by counting the cells in each well with an automated cell counter (Invitrogen). Proliferation assays were done in sextuplicate in three separate experiments.

Cell migration was assessed by performing wound healing assays coupled with measurement of wound density every two hours for $72 \mathrm{~h}$ with the Incucyte LiveCell Imaging System (Essen Bioscience). Migration assays were done in sextuplicate in two separate experiments.

\section{Generation of pancreatic cancer xenografts and assessment of tumor growth and metastases}

All animal experiments and maintenance conformed to the guidelines of the Animal Care and Use Committee of Johns Hopkins University and of the American Association of Laboratory Animal Care. Panc2.5 and Panc3.014 stable transfectants (overexpressing EYA2) and control (empty pcDNA6.2/cLumio-DEST vector) cells [10x10 cells in $200 \mu \mathrm{L}$ of $1 / 1(\mathrm{v} / \mathrm{v}) \mathrm{PBS} / \mathrm{Matrigel}]$ were injected subcutaneously into male CD1 nu/nu athymic mice (Charles River, Wilmington, MA). One week after the tumor cell injection, subcutaneous tumor volumes (V) were measured weekly with digital calipers (Fisher Scientific) and calculated using the formula $V=1 / 2\left(a b^{2}\right)$, where $a$ is the biggest and $b$ is the smallest orthogonal tumor diameter. After 3 weeks, the subcutaneous tumors from Panc2.5 stable transfectants and controls were harvested and cut into cubes of approximately $1 \mathrm{~mm}^{3}$ and orthotopic xenografts by surgical implantation were performed as described previously [42].

After 11 weeks, the mice were euthanized. The primary tumors were harvested. Each primary tumor was weighed with an analytical balance (Mettler Toledo, Switzerland). The tumor volume were measured with calipers of three orthogonal diameters ( $a, b$ and $c)$ and calculated using the formula $V=1 / 2(a b c)$. Spleen, pancreas, liver, intestine, colon, lymph node, peritoneum and lungs were inspected for grossly visible metastases and preserved in 10\% formalin solution (Sigma-Aldrich) for histology. Regional lymph nodes were harvested and a histologic section examined if lymph node metastases were suspected from macroscopic inspection.

\section{Affymetrix Exon Arrays}

Total RNA from Panc2.5 and Panc3.014 cells was extracted using mirVana miRNA Isolation Kit (Ambion, Austin, TX) per manufacturer's protocol before DNase treatment (Ambion).

The Affymetrix Exon Array ST1.0 (Affymetrix, Santa Clara, CA) was used to analyze gene expression profiles as previously described [25][43]. We are in compliance with the Minimum Information about a Microarray Experiment (MIAME) guidelines and have submitted our microarray data set to the NCBI's Gene Expression Omnibus under Accession Number GSE33282.

The presence of transcription factor binding sites within the promoters (from $2000 \mathrm{bp}$ upstream the transcription start site) of potential target genes was assessed using the MAPPER Search Engine [44]. A customized model was built by supplying a multiple sequence alignment of binding sites for EYA2 transcription factor according to the previously described consensus binding sequence GTAANYNGANAYS ( $\mathrm{N}=$ any nucleotide, $\mathrm{Y}=\mathrm{T} / \mathrm{C}$, and $\mathrm{S}=\mathrm{C} / \mathrm{G})[18,45]$.

\section{Immunoprecipitation and western blotting}

Total cellular extracts from Panc2.5- and Panc3.014EYA2-expressing and control clones (200 or $100 \mu \mathrm{g}$, respectively) were prepared using standard procedures[46] and immunoprecipitated overnight at $4{ }^{\circ} \mathrm{C}$ with either $1 \mu \mathrm{g}$ of an anti-TGFbR2 antibody or normal Rabbit IgG, as a negative control, and EZ view Red protein A Affinity Gel (Sigma), according to the manufacturer's instructions. Western blotting was performed as described previously [46]. The membrane was probed with an anti-phosphoTGFbR2 or anti-TGFbR2 antibody. Relative quantification was performed using the Gel Analyst software.

\section{Statistics}

Statistical analysis of gene expression array data was completed with Partek Genomic Suite 6.4 software. Raw Affymetrix intensity measurements of all probe sets were background corrected and normalized by the Robust Multichip Average method. Gene expression intensities were summarized by the one-step Tukey's biweight method. Survival rates were calculated by the Kaplan-Meier method, and statistical significance was examined by the log-rank test and the Cox proportional hazards regression model. One-way ANOVA analysis was performed to identify significant expression changes between EYA2-overexpressing and control pancreatic cancer cells and between control pancreatic cancer cells and the parental, non-transfected cell line. P-values of less than 0.05 were considered statistically significant. Statistical analyses were performed using SPSS version 11 (SPSS Inc., Chicago, Illinois).

\section{Grant support:}

This work was supported by NIH grants (P50CA62924), and the Michael Rolfe Foundation. 


\section{REFERENCES}

1. Vincent A, Herman J, Schulick R, Hruban RH and Goggins M. Pancreatic cancer. Lancet. 2011; 378(9791):607-620.

2. Kanda M, Matthaei H, Wu J, Hong SM, Yu J, Borges M, Hruban RH, Maitra A, Kinzler K, Vogelstein B and Goggins M. Presence of Somatic Mutations in Most Early-Stage Pancreatic Intraepithelial Neoplasia. Gastroenterology. 2012; 142:730-733.e739. Epub 2012Jan 2015 PMC3321090.

3. Jones S, Zhang X, Parsons DW, Lin JC, Leary RJ, Angenendt P, Mankoo P, Carter H, Kamiyama H, Jimeno A, Hong SM, Fu B, Lin MT, Calhoun ES, Kamiyama $\mathrm{M}$, Walter $\mathrm{K}$, et al. Core signaling pathways in human pancreatic cancers revealed by global genomic analyses. Science. 2008; 321(5897):1801-1806. Epub 2008 Sep 1804.

4. Hruban RH, Maitra A and Goggins M. Update on pancreatic intraepithelial neoplasia. Int J Clin Exp Pathol. 2008; 1(4):306-316.

5. Biankin AV, Waddell N, Kassahn KS, Gingras MC, Muthuswamy LB, Johns AL, Miller DK, Wilson PJ, Patch AM, Wu J, Chang DK, Cowley MJ, Gardiner BB, Song S, Harliwong I, Idrisoglu S, et al. Pancreatic cancer genomes reveal aberrations in axon guidance pathway genes. Nature. 2012; 491(7424):399-405.

6. Kanda M, Sadakari Y, Borges M, Topazian M, Farrell J, Syngal S, Lee J, Kamel I, Lennon AM, Knight S, Fujiwara S, Hruban RH, Canto MI and Goggins M. Mutant TP53 in Duodenal Samples of Pancreatic Juice From Patients With Pancreatic Cancer or High-Grade Dysplasia. Clinical gastroenterology and hepatology : the official clinical practice journal of the American Gastroenterological Association. 2013; 11:719-730 epub Nov 728, 2012 PMC3600161.

7. Roberts NJ, Jiao Y, Yu J, Kopelovich L, Petersen GM, Bondy ML, Gallinger S, Schwartz AG, Syngal S, Cote ML, Axilbund J, Schulick R, Ali SZ, Eshleman JR, Velculescu VE, Goggins M, et al. ATM mutations in patients with hereditary pancreatic cancer. Cancer discovery. 2012; 2(1):41-46.

8. Kim H, Saka B, Knight S, Borges M, Childs E, Klein AP, Wolfgang CL, Herman JM, Adsay V, Hruban RH and Goggins M. Having pancreatic cancer with tumoral loss of ATM and normal TP53 protein expression is associated with a poorer prognosis. Clinical cancer research : an official journal of the American Association for Cancer Research. 2014.

9. Kanda M, Knight S, Topazian M, Syngal S, Farrell JJ, Lee J, Kamel I, Lennon AM, Borges M, Young A, Fujiwara S, Seike J, Eshleman J, Hruban RH, Canto MI and Goggins M. Mutant GNAS detected in duodenal collections of secretin-stimulated pancreatic juice indicates the presence or emergence of pancreatic cysts. Gut. 2013; 62:1024-1033 epub 1028/1022/2012 PMCID: Journal in process.

10. Wu J, Matthaei H, Maitra A, Dal Molin M, Wood LD,
Eshleman JR, Goggins M, Canto MI, Schulick RD, Edil BH, Wolfgang CL, Klein AP, Diaz LA, Jr., Allen PJ, Schmidt CM, Kinzler KW, et al. Recurrent GNAS Mutations Define an Unexpected Pathway for Pancreatic Cyst Development. Science translational medicine. 2011; 3(92):92ra66.

11. Wu J, Jiao Y, Dal Molin M, Maitra A, de Wilde RF, Wood LD, Eshleman JR, Goggins MG, Wolfgang CL, Canto MI, Schulick RD, Edil BH, Choti MA, Adsay V, Klimstra DS, Offerhaus GJ, et al. Whole-exome sequencing of neoplastic cysts of the pancreas reveals recurrent mutations in components of ubiquitin-dependent pathways. Proceedings of the National Academy of Sciences of the United States of America. 2011; 108(52):21188-21193.

12. Ayars $M$ and Goggins M. (2013). Epigenetic alterations in pancreatic cancer. In: Simeone D and Maitra A, eds. Molecular Alterations of Pancreatic Cancer. (New York: Springer), pp. 185-208.

13. Vincent A, Omura N, Hong SM, Jaffe A, Eshleman J and Goggins M. Genome-Wide Analysis of Promoter Methylation Associated with Gene Expression Profile in Pancreatic Adenocarcinoma. Clinical cancer research : an official journal of the American Association for Cancer Research. 2011; 17:4341-4354.

14. Nones K, Waddell N, Song S, Patch AM, Miller D, Johns A, Wu J, Kassahn KS, Wood D, Bailey P, Fink L, Manning S, Christ AN, Nourse C, Kazakoff S, Taylor D, et al. Genome-wide DNA methylation patterns in pancreatic ductal adenocarcinoma reveal epigenetic deregulation of SLIT-ROBO, ITGA2 and MET signaling. International journal of cancer Journal international du cancer. 2014.

15. Tzatsos A, Paskaleva P, Ferrari F, Deshpande V, Stoykova S, Contino G, Wong KK, Lan F, Trojer P, Park PJ and Bardeesy N. KDM2B promotes pancreatic cancer via Polycomb-dependent and -independent transcriptional programs. The Journal of clinical investigation. 2013; 123(2):727-739.

16. Li A, Omura N, Hong SM, Vincent A, Walter K, Griffith M, Borges $\mathrm{M}$ and Goggins M. Pancreatic cancers epigenetically silence SIP1 and hypomethylate and overexpress miR$200 \mathrm{a} / 200 \mathrm{~b}$ in association with elevated circulating miR-200a and miR-200b levels. Cancer research. 2010; 70(13):5226-5237. Epub 2010 Jun 5215.

17. Jemc $J$ and Rebay I. Identification of transcriptional targets of the dual-function transcription factor/phosphatase eyes absent. Dev Biol. 2007; 310(2):416-429.

18. Jemc J and Rebay I. The eyes absent family of phosphotyrosine phosphatases: properties and roles in developmental regulation of transcription. Annu Rev Biochem. 2007; 76:513-538.

19. Krishnan N, Jeong DG, Jung SK, Ryu SE, Xiao A, Allis CD, Kim SJ and Tonks NK. Dephosphorylation of the C-terminal tyrosyl residue of the DNA damage-related histone H2A.X is mediated by the protein phosphatase eyes absent. J Biol Chem. 2009; 284(24):16066-16070. 
20. Li X, Oghi KA, Zhang J, Krones A, Bush KT, Glass CK, Nigam SK, Aggarwal AK, Maas R, Rose DW and Rosenfeld MG. Eya protein phosphatase activity regulates Six1-Dach-Eya transcriptional effects in mammalian organogenesis. Nature. 2003; 426(6964):247-254.

21. Zhang L, Yang N, Huang J, Buckanovich RJ, Liang S, Barchetti A, Vezzani C, O’Brien-Jenkins A, Wang J, Ward MR, Courreges MC, Fracchioli S, Medina A, Katsaros D, Weber BL and Coukos G. Transcriptional coactivator Drosophila eyes absent homologue 2 is up-regulated in epithelial ovarian cancer and promotes tumor growth. Cancer Res. 2005; 65(3):925-932.

22. Farabaugh SM, Micalizzi DS, Jedlicka P, Zhao R and Ford HL. Eya2 is required to mediate the pro-metastatic functions of Six 1 via the induction of TGF-beta signaling, epithelial-mesenchymal transition, and cancer stem cell properties. Oncogene. 2012; 31(5):552-562.

23. Clark SW, Fee BE and Cleveland JL. Misexpression of the eyes absent family triggers the apoptotic program. J Biol Chem. 2002; 277(5):3560-3567.

24. Zou H, Harrington JJ, Shire AM, Rego RL, Wang L, Campbell ME, Oberg AL and Ahlquist DA. Highly methylated genes in colorectal neoplasia: implications for screening. Cancer Epidemiol Biomarkers Prev. 2007; 16(12):2686-2696.

25. Omura N, Griffith M, Vincent A, Li A, Hong SM, Walter K, Borges M and Goggins M. Cyclooxygenasedeficient pancreatic cancer cells use exogenous sources of prostaglandins. Mol Cancer Res. 2010; 8(6):821-832

26. Sato N, Matsubayashi H, Abe T, Fukushima N and Goggins M. Epigenetic down-regulation of CDKN1C/p57KIP2 in pancreatic ductal neoplasms identified by gene expression profiling. Clinical cancer research : an official journal of the American Association for Cancer Research. 2005; 11(13):4681-4688.

27. O'Neill GM, Seo S, Serebriiskii IG, Lessin SR and Golemis EA. A new central scaffold for metastasis: parsing HEF1/ Cas-L/NEDD9. Cancer Res. 2007; 67(19):8975-8979.

28. Li Y, Cao H, Jiao Z, Pakala SB, Sirigiri DN, Li W, Kumar $\mathrm{R}$ and Mishra L. Carcinoembryonic antigen interacts with TGF- $\{$ beta $\}$ receptor and inhibits TGF- $\{$ beta $\}$ signaling in colorectal cancers. Cancer research. 2010; 70(20):81598168.

29. Mishima $\mathrm{N}$ and Tomarev S. Chicken Eyes absent 2 gene: isolation and expression pattern during development. Int $\mathrm{J}$ Dev Biol. 1998; 42(8):1109-1115.

30. Duncan MK, Kos L, Jenkins NA, Gilbert DJ, Copeland NG and Tomarev SI. Eyes absent: a gene family found in several metazoan phyla. Mamm Genome. 1997; 8(7):479485.

31. Zimmerman JE, Bui QT, Steingrimsson E, Nagle DL, Fu W, Genin A, Spinner NB, Copeland NG, Jenkins NA, Bucan $\mathrm{M}$ and Bonini NM. Cloning and characterization of two vertebrate homologs of the Drosophila eyes absent gene. Genome Res. 1997; 7(2):128-141.

32. Fougerousse F, Durand M, Lopez S, Suel L, Demignon J, Thornton C, Ozaki H, Kawakami K, Barbet P, Beckmann JS and Maire P. Six and Eya expression during human somitogenesis and MyoD gene family activation. J Muscle Res Cell Motil. 2002; 23(3):255-264.

33. Lobry $\mathrm{C}, \mathrm{Oh} \mathrm{P}$ and Aifantis I. Oncogenic and tumor suppressor functions of Notch in cancer: it's NOTCH what you think. The Journal of experimental medicine. 2011; 208(10):1931-1935.

34. Goggins M, Shekher M, Turnacioglu K, Yeo CJ, Hruban $\mathrm{RH}$ and Kern SE. Genetic alterations of the transforming growth factor beta receptor genes in pancreatic and biliary adenocarcinomas. Cancer research. 1998; 58(23):53295332.

35. Hahn SA, Schutte M, Hoque ATMS, Moskaluk CA, da Costa LT, Rozenblum E, Weinstein CL, Fischer A, Yeo CJ, Hruban RH and Kern SE. DPC4, a candidate tumorsuppressor gene at 18q21.1. Science. 1996; 271:350-353.

36. Infante JR and Matsubayashi H SN, Tonascia J, Klein AP, Riall TA, Yeo C, Iacobuzio-Donahue C, Goggins M. Peritumoral fibroblast SPARC expression and patient outcome with resectable pancreatic adenocarcinoma. J Clin Onc 2007; 25:319-325.

37. Merlin J, Stechly L, de Beauce S, Monte D, Leteurtre E, van Seuningen I, Huet G and Pigny P. Galectin-3 regulates MUC1 and EGFR cellular distribution and EGFR downstream pathways in pancreatic cancer cells. Oncogene. 2011; 30(22):2514-2525.

38. Burgess A, Vigneron S, Brioudes E, Labbe JC, Lorca T and Castro A. Loss of human Greatwall results in G2 arrest and multiple mitotic defects due to deregulation of the cyclin B-Cdc2/PP2A balance. Proc Natl Acad Sci U S A. 2010; 107(28):12564-12569.

39. Sato N, Fukushima N, Maitra A, Matsubayashi H, Yeo CJ, Cameron JL, Hruban RH and Goggins M. Discovery of novel targets for aberrant methylation in pancreatic carcinoma using high-throughput microarrays. Cancer research. 2003; 63(13):3735-3742.

40. Omura N, Li CP, Li A, Hong SM, Walter K, Jimeno A, Hidalgo $\mathrm{M}$ and Goggins $\mathrm{M}$. Genome-wide profiling of methylated promoters in pancreatic adenocarcinoma. Cancer Biol Ther. 2008; 7(7):1146-1156.

41. Vincent A, Perrais M, Desseyn JL, Aubert JP, Pigny P and Van Seuningen I. Epigenetic regulation (DNA methylation, histone modifications) of the $11 \mathrm{p} 15$ mucin genes (MUC2, MUC5AC, MUC5B, MUC6) in epithelial cancer cells. Oncogene. 2007; 26(45):6566-6576.

42. Feldmann G, Dhara S, Fendrich V, Bedja D, Beaty R, Mullendore M, Karikari C, Alvarez H, Iacobuzio-Donahue C, Jimeno A, Gabrielson KL, Matsui W and Maitra A. Blockade of hedgehog signaling inhibits pancreatic cancer invasion and metastases: a new paradigm for combination therapy in solid cancers. Cancer Res. 2007; 67(5):2187- 
2196.

43. Walter K, Omura N, Hong SM, Griffith M, Vincent A, Borges $\mathrm{M}$ and Goggins M. Overexpression of smoothened activates the sonic hedgehog signaling pathway in pancreatic cancer-associated fibroblasts. Clinical cancer research : an official journal of the American Association for Cancer Research. 2010; 16(6):1781-1789. Epub 2010 Mar 1789.

44. Marinescu VD, Kohane IS and Riva A. MAPPER: a search engine for the computational identification of putative transcription factor binding sites in multiple genomes. BMC Bioinformatics. 2005; 6:79.

45. Pauli T, Seimiya M, Blanco J and Gehring WJ. Identification of functional sine oculis motifs in the autoregulatory element of its own gene, in the eyeless enhancer and in the signalling gene hedgehog. Development. 2005; 132(12):2771-2782.

46. Piessen G, Jonckheere N, Vincent A, Hemon B, Ducourouble MP, Copin MC, Mariette C and Van Seuningen I. Regulation of the human mucin MUC4 by taurodeoxycholic and taurochenodeoxycholic bile acids in oesophageal cancer cells is mediated by hepatocyte nuclear factor 1alpha. Biochem J. 2007; 402(1):81-91. 